\title{
Fenótipos de resistência antimicrobiana epidemiologicamente importantes em culturas de vigilância de um serviço terciário de saúde em Aracaju-SE
}

\section{Epidemiologically relevant antimicrobial resistance phenotypes in surveillance cultures of a tertiary health service in Aracaju-SE}

\section{Francisco Lucas Rosa Amâncio'}

Izabel Karoline do Nascimento Pereira Carvalho' iD

Thatiane de Andrade Menezes" iD

Ricardo Luiz Cavalcanti de Albuquerque Junior"II (iD

Agenor Gomes dos Santos Neto"II,* (iD)

Malone Santos Pinheiro'
1 Universidade Tiradentes (Unit), Aracaju, Sergipe, Brasil

" Hospital Primavera, Aracaju, Sergipe, Brasil

III Instituto de Tecnologia e Pesquisa, Aracaju, SE, Brasil

\footnotetext{
* E-mail: agenor.biomedico@gmail.com
}

Recebido: 05 mar 2020

Aprovado: 22 jul 2020

\section{RESUMO}

Introdução: $O$ constante surgimento e a disseminação de fenótipos bacterianos de resistência a múltiplas drogas têm se tornado problemas de saúde pública em todo o mundo. Objetivo: Investigar a ocorrência de fenótipos críticos de resistência aos antimicrobianos em bactérias isoladas de culturas de vigilância. Método: Os dados microbiológicos foram coletados a partir de culturas de vigilância realizadas entre janeiro e dezembro de 2015 em um serviço terciário de saúde. Foram analisadas 1.590 culturas de vigilância utilizando o sistema VITEK 2 para identificação fenotípica e suscetibilidade aos antimicrobianos dos isolados. A detecção de B-lactamases de espectro estendido (ESBL) foi realizada por método de disco aproximação e a resistência aos carbapenêmicos e Staphylococcus aureus resistente à meticilina (MRSA) por métodos de disco difusão. A detecção de bactérias portadoras dos genes blaKPC, blaNDM e blaOXA-48 foi realizada através da reação em cadeia da polimerase in house. Resultados: Foram identificadas 201 (12,64\%) fenótipos de ESBL, 173 (11,87\%) de resistência aos carbapenêmicos e $3(0,18 \%)$ de MRSA. Foram detectadas 34 cepas portadoras de genes codificadores de carbapenemases, onde $23(67,64 \%)$ carreavam o gene blaKPC, oito $(23,53 \%)$ o blaNDM e três $(8,82 \%)$ ambos os genes. Conclusões: É um desafio controlar a disseminação de fenótipos críticos de resistência bacterianos. O predomínio de ESBL no referente hospital, assim como a ocorrência de genes codificadores de carbapenemases até então não relatados, ratificam a importância da vigilância epidemiológica e do incentivo a pesquisas subsequentes.

PALAVRAS-CHAVE: Enterobacteriaceae Produtoras de Carbapenemase; Genes Bacterianos; Infecção Hospitalar; Vigilância Epidemiológica

\begin{abstract}
Introduction: The constant emergence and spread of bacterial phenotypes of resistance to multiple drugs has become a public health problem worldwide. Objective: To investigate the occurrence of critical antimicrobial resistance phenotypes in bacteria isolated from surveillance cultures. Method: Microbiological data were collected from surveillance cultures performed between January and December 2015 at a tertiary health service. 1,590 surveillance cultures using the VITEK 2 system for phenotypic identification and susceptibility to antimicrobials of the isolates were analyzed. The detection of extended-spectrum B-lactamases (ESBL) was performed using the approach disc and carbapenem resistance method and methicillin-resistant Staphylococcus aureus (MRSA) using diffusion disc methods. The detection of bacteria carrying the blaKPC, blaNDM and blaOXA-48 genes was carried out through the polymerase chain reaction in house. Results: The analysis identified 201 (12.64\%) ESBL phenotypes, 173 (11.87\%) resistant to carbapenems and $3(0.18 \%)$ MRSA. 34 strains carrying genes encoding carbapenemases were detected, where $23(67.64 \%)$ carried the blaKPC gene, 8 (23.53\%) blaNDM and $3(8.82 \%)$ both genes. Conclusions: It is a challenge to control the spread of critical resistance bacterial phenotypes. The predominance of ESBL in the referent hospital, as well as the occurrence of genes that have not been reported for carbapenemases until then, confirm the importance of epidemiological surveillance and the encouragement of subsequent research.
\end{abstract}

KEYWORDS: Carbapenemase-producing Enterobacteriaceae; Bacterial Genes; Nosocomial Infection; Epidemiological Surveillance 


\section{INTRODUÇ̃̃O}

O surgimento e a disseminação de bactérias resistentes a múltiplas drogas (MDR) tornaram-se problemas de saúde pública em todo o mundo, por consequência do aumento do número de infecções relacionadas à assistência à saúde (IRAS) ${ }^{1}$. As bactérias são capazes de desenvolver rapidamente mecanismos que invalidam a aplicação de vários fármacos ${ }^{2,3}$.

As bactérias apresentam mecanismos de resistência aos antimicrobianos, tais como: alteração de permeabilidade da membrana celular por modificações nos canais proteicos (porinas), como ocorre para Pseudomonas aeruginosa; alteração no sítio de ligação do fármaco, no caso de Staphylococcus aureus resistente à meticilina (MRSA); bombas de efluxo que expulsam o fármaco do interior da célula bacteriana, comuns na resistência de Escherichia coli às tetraciclinas; e a produção e inativação enzimática do antimicrobiano, aplicável a Klebsiella pneumoniae Carbapenemase (KPC) e B-lactamases de espectro estendido (ESBL) que podem ocorrer na ordem Enterobacteriales s,5,6,7. $^{4}$.

MRSA, por meio da aquisição do gene mecA, codifica uma proteína adicional de ligação à penicilina (PBP) PBP2a, que possui baixa afinidade por B-lactâmicos e, portanto, resulta em resistência a fármacos dessa classe, com exceção, até o momento, das cefalosporinas ceftarolina e ceftobiprole ${ }^{8,9}$.

As ESBL são um grupo de enzimas transmitidas e codificadas por plasmídeos em bactérias Gram-negativas, capazes de degradar cefalosporinas de terceira geração e monobactâmicos ${ }^{10}$. Estas enzimas podem ser inativadas por inibidores de B-lactamases como clavulanato, sulbactam e tazobactam ${ }^{10}$.

A enzima KPC é uma carbapenemase produzida por bactérias Gram-negativas portadoras do gene blaKPC capaz de inativar toda a classe de beta-lactâmicos incluindo os carbapenêmicos. A enzima é detectada por métodos fenotípicos e confirmada por meio de biologia molecular ${ }^{11,12}$. Essa carbapenemase foi primeiramente descrita em 2001, em uma cepa de $K$. pneumoniae isolada na Carolina do Norte $^{10}$. Os primeiros relatos deste mecanismo de resistência no Brasil datam de 2009, em estudos feitos com cepas isoladas nas cidades de Recife e do Rio de Janeiro entre os anos de 2006 e $2008^{13,14}$. Estes foram seguidos de muitos outros relatos em hospitais de diferentes regiões do país ${ }^{15}$.

Conceitua-se como fenótipo crítico de resistência, a capacidade de um microrganismo inativar a principal classe de antimicrobianos clinicamente eficaz (por exemplo, KPC e MRSA) ${ }^{9,16}$. A expressão de mecanismos de resistência pode induzir a resistência a múltiplas classes de antimicrobianos de primeira escolha no tratamento de infecções graves. Assim, comumente, uma bactéria que expressa fenótipos críticos de resistência é também $M D R^{9,16}$.

Nesse contexto, é importante atentar-se ao surgimento de fenótipos críticos por meio de mecanismos de resistência antimicrobiana responsáveis por inibir os efeitos dos antimicrobianos usados no tratamento, visto que alguns destes são epidemiologicamente relevantes para o controle de infecções e para a saúde pública9.

Nesse ínterim, a partir do registro de casos de pacientes infectados com KPC em agosto de 2015 em um serviço terciário de saúde em Aracaju, foi investigada a ocorrência de fenótipos críticos de resistência aos antimicrobianos em bactérias isoladas de culturas de vigilância.

\section{MÉTODO}

\section{Tipo de estudo}

Trata-se de um estudo retrospectivo, descritivo, transversal, de abordagem quantitativa com a finalidade de observar a ocorrência de fenótipos bacterianos críticos isolados a partir de culturas de vigilância. A amostra de estudo constou de informações sobre o microrganismo e seu perfil de resistência oriundos de relatórios de controle de infecção hospitalar advindos de arquivos de culturas de vigilância de um serviço terciário de saúde, localizado na cidade de Aracaju, no estado de Sergipe, Brasil, entre janeiro e dezembro de 2015. Os dados contidos foram obtidos a partir de fichas impressas e assinadas pelo responsável do laboratório de microbiologia da referida instituição.

Foram investigados apenas casos de fenótipos bacterianos críticos. As variáveis analisadas foram o tipo de amostra de fenótipos críticos de interesse epidemiológico: MRSA, ESBL e as carbapenemases KPC, New Delhi Metalo-B-lactamase (NDM) e OXA-48.

\section{Amostras}

No período estudado, um total de 1.590 culturas de vigilância provenientes de colonização foi analisado. Os dados foram adquiridos a partir da disponibilidade de resultados oriundos das culturas de vigilâncias distribuídos equitativamente entre swabs retais, nasais e axilares coletados de pacientes em leitos de unidades de tratamento intensivo (UTI), clínica, cirúrgica e enfermarias de urgência. A coleta do material foi realizada conforme os protocolos de controle de infecção hospitalar da instituição.

\section{Análise das amostras}

Após a cultura para isolamento, os microrganismos foram analisados por meio do equipamento VITEK $^{\oplus} 2$ bioMérieux, que promove identificação fenotípica e realização do teste de sensibilidade aos antimicrobianos (TSA) por método semiquantitativo. Foi analisado o perfil de sensibilidade aos antimicrobianos das espécies identificadas para posterior realização dos testes de detecção de fenótipos críticos de acordo com os procedimentos padrões do laboratório de microbiologia clínica da instituição.

\section{Detecção de fenótipos críticos}

Após identificação e análise do TSA, os microrganismos foram submetidos a testes fenotípicos para confirmação. Para a detecção do fenótipo ESBL em microrganismos Gram-negativos resistentes 
às cefalosporinas, utilizou-se o teste do disco combinado (TDC) com discos contendo a cefalosporina isoladamente (cefotaxima, ceftazidima e cefepima) e em combinação com o ácido clavulânico ${ }^{17,18}$. O fenótipo presente em MRSA foi detectado a partir do método de disco-difusão utilizando a cefoxitina em isolados de S. aureus. A triagem inicial para detecção de carbapenemases foi realizada por meio da seleção de microrganismos resistentes aos carbapenêmicos (meropenem, imipenem e ertapenem) e posterior confirmação por métodos moleculares ${ }^{17,18}$.

\section{Detecção genotípica de carbapenemases}

Para a confirmação desse fenótipo, as linhagens bacterianas Gram-negativas resistentes aos carbapenêmicos foram encaminhadas pelo serviço a um laboratório de apoio da Fundação Oswaldo Cruz (Fiocruz) no Rio de Janeiro, onde, por meio da metodologia de reação em cadeia da polimerase (PCR) in house, foram pesquisados os genes codificadores de carbapenemases blaKPC, blaNDM e blaOXA-48 ${ }^{10,19,20}$. Foram considerados fenótipos produtores de carbapenemase a presença de um ou mais genes codificadores. As bactérias que não apresentaram os genes pesquisados, foram consideradas como linhagens resistentes aos carbapenêmicos pela presença de outros mecanismos de resistência.

\section{Análise dos dados}

Os dados extraídos foram armazenados em planilhas do Excel $\odot$ versão 2019. Por meio da estatística descritiva os dados foram apresentados utilizando de distribuição de frequência relativa e absoluta. A figura com a frequência das variáveis categorizadas foi obtida utilizando os softwares GraphPad Prism@ 8 e Origin@ 2018.

\section{Aspetos éticos}

Esse trabalho foi aprovado pelo Comitê de Ética em Pesquisa (CEP) da Universidade Tiradentes sob o parecer número
3.801.168 de acordo com a Resolução do Conselho Nacional de Saúde $n^{\circ}$ 466, de 12 de dezembro de 2012.

\section{RESULTADOS}

Das 1.590 culturas de vigilância analisadas, 377 apresentaram microrganismos com fenótipos críticos de resistência. Os swabs retais demonstraram o maior percentual de fenótipos detectados, totalizando $210(55,70 \%)$ dos isolados, ao passo que 92 $(24,40 \%)$ nos swabs axilares e $75(19,90 \%)$ nos swabs nasais. Não foi possível discriminar os locais da instituição onde foram coletados os swabs devido à falta de informações no banco de dados.

Em relação ao total de amostras analisado, foram identificados 201 (12,64\%) fenótipos de ESBL, $173(11,87 \%)$ resistentes aos carbapenêmicos e três $(0,18 \%)$ MRSA.

Atentando-se aos fenótipos críticos, é importante observar a presença de ESBL e a resistência aos carbapenêmicos com frequência variável em todos os meses de análise. Todavia, o mês de dezembro registrou maior número destes fenótipos. Carbapenemases foram detectadas de agosto a dezembro, e setembro foi o mês com maior número de casos. MRSA foi o fenótipo com menor número de registros, aparecendo somente nos meses de março, agosto e setembro (Figura).

Entre os fenótipos críticos identificados no presente estudo, predominaram 120 (31,83\%) isolados de $E$. coli e $81(21,50 \%)$ de $K$. pneumoniae como produtores de ESBL, e $139(36,87 \%)$ isolados de $P$. aeruginosa resistentes aos carbapenêmicos. Todas as 34 $(9,01 \%)$ Enterobacteriaceae portadoras de genes para carbapenemase pertenciam à espécie $K$. pneumoniae, onde testes moleculares demonstraram que $23(67,64 \%)$ apresentaram o gene blaKPC, oito $(23,53 \%)$ apresentaram blaNDM e três $(8,82 \%)$ apresentaram ambos os genes. 0 gene blaOXA-48 não foi encontrado em nenhum dos isolados bacterianos. Foram também identificados no estudo três $(0,79 \%)$ MRSA.

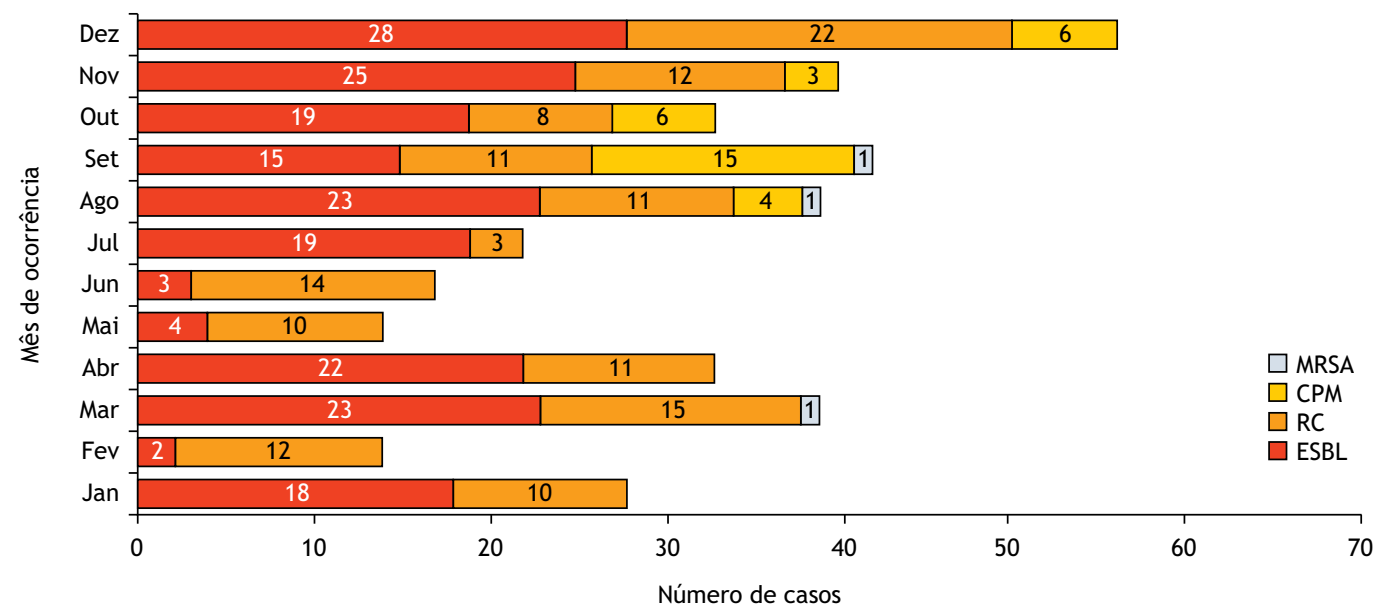

Fonte: Elaborada pelos autores, 2020.

ESBL: B-lactamase de espectro estendido; RC: resistências aos carbapenêmicos; CPM: carbapenemases; MRSA: Staphylococcus aureus resistente à meticilina.

Figura. Ocorrência de fenótipos bacterianos críticos detectados em culturas de vigilância no período de janeiro a dezembro de 2015 em um serviço terciário de saúde em Aracaju-SE. 


\section{DISCUSSÃO}

A ESBL foi inicialmente documentada na Europa, com posterior disseminação a nível mundial, estando atualmente presente também no território brasileiro com diversas variantes já detectadas ${ }^{21,22}$. 0 presente estudo evidenciou o predomínio de fenótipos produtores de ESBL e corrobora com as pesquisas já realizadas que revelaram prevalência de ESBL em culturas de vigilância ${ }^{23,24,25}$.

A relevância clínica da ESBL está relacionada à redução da opção terapêutica limitada aos carbapenêmicos como a classe de escolha para o tratamento de infecções por este fenótipo ${ }^{26,27,28}$. Esta enzima é codificada por genes presentes em plasmídeos, segmentos genéticos facilmente transferíveis entre as bactérias, favorecendo a disseminação de resistência tanto no âmbito hospitalar quanto na comunidade. Também é comum o carregamento de genes conferindo resistência a outras classes antimicrobianas ${ }^{26,27}$.

O fenótipo representado pelo MRSA foi o menos evidenciado quando comparado aos demais no período de estudo. Este microrganismo é relatado como importante patógeno hospitalar, exibindo alta virulência e produção de toxinas, causando infecções em tecidos moles e pele ${ }^{29}$. Pacientes que apresentam colonização das narinas por este agente são os principais contribuintes para sua disseminação ${ }^{30}$. As cepas de MRSA são resistentes aos fármacos B-lactâmicos, incluindo carbapenêmicos, e elevam a probabilidade de óbito em até cinco vezes quando comparado a $S$. aureus sensíveis à meticilina ${ }^{31}$.

Com relação à resistência aos carbapenêmicos e à produção de carbapenemases, esta classe de enzimas tem ganhado constante atenção pelo seu impacto clínico, uma vez que confere resistência a todos os agentes B-lactâmicos, reduzindo as opções terapêuticas e impondo grandes esforços para seu controle e prevenção, dificultados pela rápida transmissão de genes plasmidiais codificadores dessas enzimas. Maior destaque é dado para as enzimas KPC e NDM, disseminadas mundialmente $\mathrm{e}^{14,29,32}$. Pode-se observar neste estudo que $8,74 \%$ das amostras analisadas apresentavam espécies resistentes aos carbapenêmicos e 3,13\% apresentaram genes codificadores de carbapenemases, dado relevante para o controle de disseminação e infecção hospitalar.
Estudos epidemiológicos que objetivaram a pesquisa de genes codificadores de carbapenemases demonstraram predomínio do gene blaKPC $33,32,34$ e em menor escala dos genes blaNDM e blaOXA-483732,35. 0 presente trabalho coaduna com os estudos referidos e também demonstrou o gene blaKPC como predominante e secundariamente o gene blaNDM, não sendo evidenciada a presença do blaOXA- $48^{35}$.

O primeiro achado de KPC no serviço terciário objetivado neste estudo ocorreu em agosto de 2015, caracterizando-se como surto, posto que não havia relato prévio desse fenótipo. Segundo a Agência Nacional de Vigilância Sanitária ${ }^{34}$, surto é caracterizado quando há aumento acima do esperado na ocorrência de casos de evento ou doença em uma área ou entre um grupo específico de pessoas, em determinado período.

Para controle e prevenção da ocorrência e disseminação de bactérias carreadoras de genes de resistência, medidas de controle devem ser reforçadas e estabelecidas. Na comunidade, as práticas de sanitização e prevenção de doenças são cruciais para a diminuição do surgimento de infecções. No ambiente hospitalar, faz-se necessário a aplicação de políticas educacionais, a implementação de programas de gerenciamento quanto ao uso de antimicrobianos e o controle de infecção hospitalar, com o monitoramento e emissão de relatórios sobre infecções hospitalares por fenótipos críticos de resistência ${ }^{36}$.

\section{CONCLUSÕES}

As culturas de vigilância objetivadas permitiram identificar importantes fenótipos críticos de resistência aos antimicrobianos com predomínio de ESBL, frequente em serviços terciários de saúde.

Digna de nota foi a detecção de genes produtores de carbapenemases em linhagens de K. pneumoniae, haja vista esse fenótipo, até então, não ter sido relatado no serviço em questão.

A aplicação deste modelo de estudo em outros serviços terciários de saúde do estado de Sergipe faz-se necessária para evidenciar a realidade epidemiológica quanto a pesquisa de fenótipo KPC, ratificando a importância da vigilância epidemiológica.

\section{REFERÊNCIAS}

1. Chang HH, Cohen T, Grad YH, Hanage WP, O’Brien TF, Lipsitch M. Origin and proliferation of multiple-drug resistance in bacterial pathogens. Microbiol Mol Bio Rev. 2015;79(1):101-16 https://doi.org/10.1128/MMBR.00039-14

2. Ibargüen-Mondragón E, Romero-Leiton JP, Esteva L, Cerón MG, Hidalgo-Bonilla S. Stability and periodic solutions for a model of bacterial resistance to antibiotics caused by mutations and plasmids. Appl Math Model. 2019;76:238-51. https://doi.org/10.1016/j.apm.2019.06.017

3. Fernandes LS. Resistência bacteriana aos betalactâmicos por mecanismo enzimático: uma revisão de literatura com enfoques nas betalactamases de espectro estendido [monografia]. Campina Grande: Universidade Estadual da Paraíba; 2014.

4. McGinigle KL, Gourlay ML, Buchanan IB. The use of active surveillance cultures in adult intensive care units to reduce methicillin-resistant Staphylococcus aureus-related morbidity, mortality, and costs: a systematic review. Clin Infect Dis. 2008;46(11):1717-25. https://doi.org/10.1086/587901

5. Alvarez C, Labarca J, Salles M. Prevention strategies for methicillin-resistant Staphylococcus aureus (MRSA) in Latin America. Braz J Infect Dis. 2010;14(supl.2):107-18. https://doi.org/10.1590/S1413-86702010000800006 
6. Lima MRS, Soares NS, Mascarenhas MDM, Amaral EJLS. Intervenção em surto de Klebsiella pneumoniae produtora de betalactamase de espectro expandido (ESBL) em unidade de terapia intensiva neonatal em Teresina, Piauí, 2010-2011. Epidemiol Serv Saude. 2014;23(1):177-82. https://doi.org/10.5123/S1679-49742014000100017

7. Agência Nacional de Vigilância Sanitária - Anvisa. Resistência microbiana: mecanismos e impacto clínico. Brasília: Agência Nacional de Vigilância Sanitária; 2007.

8. Llarrull LI, Fisher JF, Mobashery S. Molecular basis and phenotype of methicillin resistance in Staphylococcus aureus and insights into new beta-lactams that meet the challenge. Antimicrob Agents Chemother. 2009;53(10):4051-63 . https://doi.org/10.1128/AAC.00084-09

9. Comitê Brasileiro de Testes de Sensibilidade aos Antimicrobianos - BrCAST. Orientações do EUCAST para a detecção de mecanismos de resistência e resistências específicas de importância clínica e/ou epidemiológica: versão 2.0. Brasília: Comitê Brasileiro de Testes de Sensibilidade aos Antimicrobianos; 2017.

10. Yigit $H$, Queenan AM, Anderson GJ, Domenech-Sanchez A, Biddle JW, Steward CD et al. Novel carbapenem-hydrolyzing betalactamase, KPC-1, from a carbapenem-resistant strain of Klebsiella pneumoniae. Antimicrob Agents Chemother. 2001;45(4):1151-61. https://doi.org/10.1128/AAC.45.4.1151-1161.2001

11. Steward CD, Wallace D, Hubert SK, Lawton R, Fridkin SK, Gaynes RP et al. Ability of laboratories to detect emerging antimicrobial resistance in nosocomial pathogens: a survey of project Icare laboratories. Diagn Microbiol Infect Dis. 2000;38(1):59-67. https://doi.org/10.1016/s0732-8893(00)00161-9

12. Moland ES, Hanson ND, Herrera VL, Black JA, Lockhart TJ, Hossain A et al. Plasmid-mediated, carbapenem-hydrolysing beta-lactamase, KPC-2, in Klebsiella pneumoniae isolates. J Antimicrob Chemother. 2003;51(3):711-4. https://doi.org/10.1093/jac/dkg124

13. Peirano G, Seki LM, Passos VLV, Pinto MCFG, Guerra LR, Asensi MD. Carbapenem-hydrolysing beta-lactamase KPC-2 in Klebsiella pneumoniae isolated in Rio de Janeiro, Brazil. J Antimicrob Chemother. 2009;63(2):265-8. https://doi.org/10.1093/jac/dkn484

14. Monteiro JAF, Santos MD, Asensi MG, Peirano G, Gales CA. First report of KPC-2-producing Klebsiella pneumoniae strains in Brazil. Antimicrob Agents Chemother. 2009;53(1):333-4. https://doi.org/10.1093/jac/dkn484

15. Bonelli RR, Moreira BM, Picão RC. Antimicrobial resistance among Enterobacteriaceae in South America: history, current dissemination status and associated socioeconomic factors. Drug Resist Updat. 2014;17(1-2):24-36. https://doi.org/10.1016/j.drup.2014.02.001

16. US Centers for Disease Control - CDC. Antibiotic resistance threats in the United States, 2019. Atlanta: US Centers for Disease Control; 2019.
17. Clinical and Laboratory Standards Institute - CLSI. M100 performance standards for antimicrobial susceptibility testing. Wayne: Clinical and Laboratory Standards Institute; 2009

18. European Committee on Antimicrobial Susceptibility Testing - EUCAST. EUCAST guidelines for detection of resistance mechanisms and specific resistances of clinicaland/or epidemiological importance. Växjö: European Committee on Antimicrobial Susceptibility Testing; 2013.

19. Nordmann P, Poirel L, Walsh TR, Livermore DM. The emerging NDM carbapenemases. Trends Microbiol. 2011;19(12):58895 https://doi.org/10.1016/j.tim.2011.09.005

20. Poirel L, Naas T, Nordmann P. Diversity, epidemiology, and genetics of class $D$ betalactamases. Antimicrob Agents Chemother. 2010;54(1):24-38. https://doi.org/10.1128/AAC.01512-08

21. Tansarli GS, Poulikakos P, Kapaskelis A, Falagas ME. Proportion of extended-spectrum B-lactamase (ESBL)-producing isolates among Enterobacteriaceae in Africa: evaluation of the evidence-systematic review. J Antimicrob Chemother. 2014;69(5):1177-84. https://doi.org/10.1093/jac/dkt500

22. Silva KC, Lincopan N. Epidemiologia das betalactamases de espectro estendido no Brasil: impacto clínico e implicações para o agronegócio. J Bras Patol Med Lab. 2012;48(2):91-9. https://doi.org/10.1590/1676-24442012000200004

23. Flores C, Romão CMCPA, Bianco K, Miranda CC, Breves A, Souza APS et al. Detecção de genes de resistência a antimicrobianos em Klebsiella pneumoniae produtoras de betalactamases e carbapenemases por culturas de vigilância de pacientes em uma unidade de terapia intensiva no Rio de Janeiro, Brasil. J Bras Patol Med Lab. 2016;52(5):284-92. https://doi.org/10.5935/1676-2444.20160049

24. Cassettari VC, Silveira IR, Balsamo AC, Franco F. Surto em berçário por Klebsiella pneumoniae produtora de beta-lactamase de espectro estendido atribuído à colonização de profissional de saúde portador de onicomicose. J Pediatr. 2006;82(4):313-16. https://doi.org/10.2223/JPED.1519

25. Soares JHR, Mendes PBS, Tacla MTGM, Lopes GK. Identificação microbiológica e perfil de resistência a antimicrobianos em crianças hospitalizadas. Rev Bras Enfer Ped. 2017;17(2):57-63.

26. Denisuik AJ, Lagacé-Wiens PR, Pitout JD, Mulvey MR, Simner PJ, Tailor $\mathrm{F}$ et al. Molecular epidemiology of extended-spectrum B-lactamase-, AmpC B-lactamase- and carbapenemase-producing Escherichia coli and Klebsiella pneumoniae isolated from canadian hospitals over a 5 year period: Canward 2007-11. J Antimicrob Chemother. 2013;68(supl.1):57-65. https://doi.org/10.1093/jac/dkt027

27. Silva KC, Lincopan N. Epidemiologia das betalactamases de espectro estendido no Brasil: impacto clínico e implicações para o agronegócio. J Bras Patol Med Lab. 2012;48(2):91-9. https://doi.org/10.1590/1676-24442012000200004

28. Rossi F, Andreazzi DB. Resistência bacteriana: interpretando o antibiograma. São Paulo: Atheneu; 2005. 
29. Moran MC, Cahill MP, Brewer MG, Yoshida T, Knowlden S, Perez-Nazario $\mathrm{N}$ et al. Staphylococcal virulence factors on the skin of atopic dermatitis patients. Msphere. 2019;4(6):1-7. https://doi.org/10.1128/mSphere.00616-19

30. Almeida LC, Pimenta-Rodrigues MV, Moris DV, Fortaleza CMCB, Cunha MLRS. Avaliação fenotípica e genotípica do perfil de resistência de amostras de Staphylococcus aureus isoladas de culturas clínicas e de vigilância de um hospital de ensino brasileiro. Colloq Vitae. 2012;4(2):68-78. https://doi.org/10.5747/cv.2012.v004.n2.v063c

31. Ribeiro IF, Silva SFR, Silva SL, Ribeiro TR, Rocha MMNP, Stolp AMV. Identificação de Staphylococcus aureus e Staphylococcus aureus resistente à meticilina em estudantes universitários. Rev Cienc Farm Basica Apl. 2014;35(2):299-302.

32. Rozales FP, Ribeiro VB, Magagnin CM, Pagano M, Lutz L, Falci DR et al. Emergence of NDM-1-producing
Enterobacteriaceae in Porto Alegre, Brazil. Int J Infect Dis. 2014;25:79-81. https://doi.org/10.1016/j.ijid.2014.01.005

33. Alves AP, Behar PRP. Infecções hospitalares por enterobactérias produtoras de KPC em um hospital terciário do sul do Brasil. Rev Amrigs. 2013;57(3):213-8.

34. Agência Nacional de Vigilância Sanitária - Anvisa. Segurança do paciente e qualidade em serviços de saúde: medidas de prevenção de infecção relacionada à assistência à saúde. Brasília: Agência Nacional de Vigilância Sanitária; 2013.

35. Aires CAM, Rocha-de-Souza CM, Timm LN, Pereira PS, Carvalho-Assef APD, Asensi MD. Early detection of OXA-370-producing Klebsiella pneumoniae ST17 co-harboring bla $a_{\text {CTX-M-8 }}$ in Brazil. Diagn Microbiol Infect Dis. 2016;86(4):434-6. https://doi.org/10.1016/j.diagmicrobio.2016.09.007

36. Agencia Nacional de Vigilância Sanitária - Anvisa. Anvisa intensifica controle de infecção em serviços de saúde. Rev Saúde Pública. 2004;38(3):475-8. https://doi.org/10.1590/S0034-89102004000300022

\section{Contribuição dos Autores}

Amâncio FLR, Carvalho IKNP - Concepção, planejamento (desenho do estudo), análise, interpretação dos dados e redação do trabalho. Menezes TA, Albuquerque Junior RLC - Aquisição, análise e interpretação dos dados. Pinheiro MS, Santos-Neto AG - Análise, interpretação dos dados e redação do trabalho. Todos os autores aprovaram a versão final do trabalho.

\section{Conflito de Interesse}

Os autores informam não haver qualquer potencial conflito de interesse com pares e instituições, políticos ou financeiros deste estudo.

Licença CC BY-NC atribuição não comercial. Com essa licença é permitido acessar, baixar (download), copiar, imprimir, compartilhar, reutilizar e distribuir os artigos, desde que para uso não comercial e com a citação da fonte, conferindo os devidos créditos de autoria e menção à Visa em Debate. Nesses casos, nenhuma permissão é necessária por parte dos autores ou dos editores. 